\title{
High-Performance Photodetectors Based on Lateral Monolayer MoS2/WS2 Heterojunctions
}

\section{Caihong Li ( $\nabla$ caihong_li@yahoo.com )}

the Institute of Fundamental and Frontier Science, University of Electronic Science and Technology of China https://orcid.org/0000-0002-9844-4333

Juntong Zhu

Soochow University

\section{Wen Du}

University of Electronic Science and Technology of China

\section{Yixuan Huang}

University of Electronic Science and Technology of China

Hao Xu

University of Electronic Science and Technology of China

\section{Zhengang Zhai}

36th Research Institute of China Electronics Technology Group Corporation

\section{Guifu Zou}

Soochow University

\section{Research Article}

Keywords: lateral monolayer heterostructure, MoS2/WS2 heterojunction, photodetector, sharp interface

Posted Date: July 9th, 2021

DOI: https://doi.org/10.21203/rs.3.rs-694892/v1

License: (c) (i) This work is licensed under a Creative Commons Attribution 4.0 International License.

Read Full License

Version of Record: A version of this preprint was published at Nanoscale Research Letters on July 31 st, 2021. See the published version at https://doi.org/10.1186/s11671-021-03581-4. 


\title{
High-Performance Photodetectors Based on Lateral Monolayer $\mathrm{MoS}_{2} / \mathrm{WS}_{2}$ Heterojunctions
}

\author{
Caihong $\mathrm{Li}^{1}$, Juntong $\mathrm{Zhu}^{2}$, Wen $\mathrm{Du}^{1}$, Yixuan Huang ${ }^{1}$, Hao Xu ${ }^{1,3,4 *}$, Zhengang Zhai ${ }^{5}$, Guifu Zou ${ }^{2 *}$
}

\begin{abstract}
Monolayer transition metal dichalcogenides (TMDs) show promising potential for next-generation optoelectronics due to excellent light capturing and photodetection capabilities. Photodetectors, as important components of sensing, imaging and communication systems, are able to perceive and convert optical signals to electrical signals. Herein, the large-area and high-quality lateral monolayer $\mathrm{MoS}_{2} / \mathrm{WS}_{2}$ heterojunctions were synthesized via the one-step liquid-phase chemical vapor deposition (CVD) approach. Systematic characterization measurements have verified good uniformity and sharp interfaces of the channel materials. As a result, the photodetectors enhanced by the photogating effect can deliver competitive performance, including responsivity of $\sim 567.6 \mathrm{~A} / \mathrm{W}$ and detectivity of $\sim 7.17 \times 10^{11} \mathrm{Jones}$. In addition, the $1 / \mathrm{f}$ noise obtained from the current power spectrum is adverse to the development of photodetectors, which is considered as originating from charge carrier trapping/detrapping. Therefore, this work may contribute to efficient optoelectronic devices based on lateral monolayer TMD heterostructures.
\end{abstract}

Keywords: lateral monolayer heterostructure, $\mathrm{MoS}_{2} / \mathrm{WS}_{2}$ heterojunction, photodetector, sharp interface

\section{Introduction}

Considering the almost half-a-trillion-dollar

semiconductor-chip market, two-dimensional (2D)

materials are currently one of the most feasible and

*Correspondence: hao.xu.15@uestc.edu.cn; guifuzou@suda.edu.cn

${ }^{1}$ Institute of Fundamental and Frontier Sciences, University of Electronic Science and Technology of China, Chengdu 610054, P. R. China.

${ }^{2}$ the College of Energy, Soochow Institute for Energy and Materials Innovations, and Key Laboratory of Advanced Carbon Materials and Wearable Energy Technologies of Jiangsu Province, Soochow University, Suzhou 215006, China.

${ }^{3}$ the State Key Laboratory of Electronic Thin Films and Integrated Devices, University of Electronic Science and Technology of China, Chengdu 610054, P. R. China

${ }^{4}$ School of Physics, University of Electronic Science and Technology of China, Chengdu 610054, P. R. China.

${ }^{5}$ the 36th Research Institute of China Electronics Technology Group Corporation, Jiaxing, 314033, P. R. China. promising candidates for extending Moore's law [1-

5]. As a representative member of the 2D family, transition metal dichalcogenides (TMDs) have been intensively studied due to their distinctive optoelectronic properties and potential applications [6-12] in photodetection and light-emitting devices $[13,14]$. Notably, the tunable bandgap, high carrier mobility, high optical absorption and atomically thin thickness, making TMDs appropriate channel 
materials for photodetectors, play a crucial role in optoelectronic or electronic devices $[15,16]$. Although crystal defects in TMDs giving rise to the carrier trapping effect can result in high photosensitivity, they can unavoidably lead to slow response speed yet [17]. Combining respective superiorities and showing unique electronic transport at the junction, TMDs heterostructures either lateral stitching or vertical stacking are presented [18]. Such heterostructures can tailor intrinsic electronic properties and improve the optical absorption [19], showing emerging and designable features $[13,20]$. For example, the builtin electrical field [21] or energy level difference [22] induced by TMD heterostructures should accelerate photocarrier separation, suppress photocarrier recombination $[17,23]$ and lower dark current [24] as well, which is beneficial for achieving high-performance photodetection. Besides, Wang's group [25] has certified suppressed electron-hole (e-h) recombination in lateral heterostructures. As previously reported, the lateral heterostructures showed higher carrier mobility [26] whereas the vertical heterostructures usually increased the photoactive area [23] and/or enhanced current drive per area [27]. Moreover, the in-plane interfaces of lateral heterostructures showed stronger emission intensity than both sides [14]. However, the suppressed photoluminescence (PL) emission could be observed in the vertical hetero-interface because of the reduced direct radiative recombination [28]. Additionally, both lateral and vertical TMDs heterostructures make it possible to create new excitonic transitions [14].

In terms of crystal lattice quality, $\mathrm{MoX}_{2} / \mathrm{WX}_{2}$ $(\mathrm{X}=\mathrm{S}$, Se or $\mathrm{Te})$ lateral heterojunctions could induce structural defects scarcely due to their similar honeycomb-like [29, 30] configuration and lattice parameters [30]. In addition, this kind of heterojunction can form type-II band alignment generally, which is desirable for high-efficiency photodetection $[28,30,31]$. According to the former work, lateral monolayer $\mathrm{MoS}_{2} / \mathrm{WS}_{2}$ heterojunction preferred to exhibit type-II band alignment with the valence band maximum (VBM) localized at $\mathrm{WS}_{2}$ and the conduction band minimum $(\mathrm{CBM})$ at $\mathrm{MoS}_{2}[28,30]$. For instance, Wu's group have further reported that the VBM and CBM of $\mathrm{MoS}_{2}$ are $0.39 \mathrm{eV}$ and $0.35 \mathrm{eV}$ lower than that of $\mathrm{WS}_{2}$, respectively [30]. Furthermore, the band 
offset between $\mathrm{MoS}_{2}$ and $\mathrm{WS}_{2}$ determining the band alignment could be estimated via their different dorbital positions of Mo and W [30]. Vertical heterostructures can be prepared by mechanical transfer and stack, whereas lateral ones can be only achieved by growth methods [14]. Furthermore, vertical heterostructures, as previously reported, cannot be precise control and it is easily contaminated at the interfaces between layers [29]. Luckily, the lateral heterostructures can be synthesized by one-step method to reduce contaminations [24]. Nowadays the growth of largearea and high-quality lateral monolayer TMDs heterostructures has remained a great challenge [32]. Hence, high-quality and large-area lateral TMDs heterojunctions are significant and desired for the development of high-performance photodetectors.

Here, the lateral monolayer $\mathrm{MoS}_{2} / \mathrm{WS}_{2}$ heterojunctions with sharp interfaces and good uniformity via one-step liquid-phase CVD method are prepared and photodetectors are fabricated based on these heterostructures. The presented photodetectors can deliver high-performance responsivity and detectivity of $567.6 \mathrm{~A} / \mathrm{W}$ and
$7.17 \times 10^{11}$ Jones, respectively. This work demonstrates lateral monolayer $\mathrm{MoS}_{2} / \mathrm{WS}_{2}$ heterojunctions can serve as qualified candidates for next-generation optoelectronic applications.

\section{Methods}

\section{Heterostructure synthesis}

$0.05 \mathrm{~g}$ sodium tungstate, $0.5 \mathrm{~g}$ ammonium molybdate and $0.12 \mathrm{~g} \mathrm{NaOH}$ (or $\mathrm{KOH}$ ) particles were dissolved in $10 \mathrm{~mL}$ of deionized (DI) water to obtain precursor solution. The growth substrates (sapphire) were treated by piranha solution to improve the surface hydrophilia, and then the precursor solution was evenly spin-coated onto clean sapphire substrates. After that, the precursor covered sapphire and sulfur were placed on the heating center and upstream of a quartz tube, respectively. The heating center was ramped up to $700{ }^{\circ} \mathrm{C}$ in $40 \mathrm{~min}$ and maintained for $10 \mathrm{~min}$ to grow $\mathrm{MoS}_{2}-\mathrm{OH}$ bilayers. Finally, the carrier gas was changed from $\mathrm{Ar}$ to $\mathrm{Ar} / \mathrm{H}_{2}\left(5 \% \mathrm{H}_{2}\right)$, and the heating center heated to $780{ }^{\circ} \mathrm{C}$ within $10 \mathrm{~min}$ and kept for 10 min to allow $\mathrm{WS}_{2}$ to grow along the edges of $\mathrm{MoS}_{2}-\mathrm{OH}$ bilayers, forming $\mathrm{MoS}_{2} / \mathrm{WS}_{2}$ lateral heterostructures.

\section{Transfer process}

We used the polystyrene (PS)-assisted method to transfer $\mathrm{WS}_{2} / \mathrm{MoS}_{2}$ lateral heterostructures from 
sapphire to $\mathrm{SiO}_{2} / \mathrm{Si}$ substrates. The PS solution $(9 \mathrm{~g}$ of PS was dissolved in $100 \mathrm{~mL}$ of toluene) is first spin-coated on the heterostructures with $3500 \mathrm{rpm}$ for $60 \mathrm{~s}$, then the sample is baked at $90{ }^{\circ} \mathrm{C}$ for 10 min to eliminate toluene. After that, the $\mathrm{WS}_{2} / \mathrm{MoS}_{2}-$ PS film is obtained by a water droplet, and the floating $\mathrm{WS}_{2} / \mathrm{MoS}_{2}-\mathrm{PS}$ film is then dredged up with a clean $\mathrm{SiO}_{2} / \mathrm{Si}$ substrate. The $\mathrm{WS}_{2} / \mathrm{MoS}_{2}-\mathrm{PS}-$ $\mathrm{SiO}_{2} / \mathrm{Si}$ sample is baked at $80{ }^{\circ} \mathrm{C}$ for $1 \mathrm{~h}$ and then at $150{ }^{\circ} \mathrm{C}$ for $30 \mathrm{~min}$ to spread the polymer to eliminate possible wrinkles. Finally, the PS film is removed by rinsing with toluene several times to get $\mathrm{WS}_{2} / \mathrm{MoS}_{2}-\mathrm{SiO}_{2} / \mathrm{Si}$ samples.

\section{Device fabrication}

The standard electron beam lithography (EBL) was used to define the markers and electrode patterns on the as-grown lateral monolayer $\mathrm{MoS}_{2} / \mathrm{WS}_{2}$ heterojunctions. The Ti/Au electrodes $(10 \mathrm{~nm} / 100$ $\mathrm{nm}$ ) were evaporated on the channel and lifted off in acetone. The device was thermal annealed at 400 ${ }^{\circ} \mathrm{C}$ for 2 hours in vacuum and cooled down to room temperature rapidly.

\section{Material characterization}

The optical images were captured with in the stitched interface marked 3 in Fig. 1(a), OLYMPUS microscope (LV100ND). The Raman, indicating two materials form at the interface. In PL and AFM mapping images were measured with a Raman-AFM confocal spectrometer (Witec, alpha300 RA) with a laser of $532 \mathrm{~nm}$.

\section{Device characterization}

The optoelectronic properties of the photodetectors were measured with the SemiProbe probe station and a semiconductor parameter analyzer (Keithley 4200) and Platform Design Automation (PDA, FSPro). Different wavelength lasers as the light sources were used to measure the photoresponse of the photodetectors. Different laser densities were determined with an irradiatometer.

\section{Results and discussion}

Fig.1(a) shows the optical image of the CVD-grown lateral monolayer heterostructure, illustrated by the optical contrast. The corresponding Raman spectra obtained from the different positions marked 1 and 2 in Fig. 1(a) confirm the configuration of the inner $\mathrm{MoS}_{2}\left(385.5 \mathrm{~cm}^{-1}\right.$ and $\left.405.3 \mathrm{~cm}^{-1}\right)$ and outer $\mathrm{WS}_{2}$ $\left(351.5 \mathrm{~cm}^{-1}\right.$ and $\left.416.5 \mathrm{~cm}^{-1}\right)$ in Fig. 1(b) [26]. High crystal quality of $\mathrm{MoS}_{2}$ and $\mathrm{WS}_{2}$ are implied because no oxidation peak observed in the corresponding Raman spectra [33]. Especially, the eigen-peaks of $\mathrm{MoS}_{2}$ and $\mathrm{WS}_{2}$ both were observed addition, the frequency difference between the $E_{2 g}$ 
mode and $\mathrm{A}_{1 \mathrm{~g}}$ mode of $\mathrm{MoS}_{2}$ is $19.8 \mathrm{~cm}^{-1}$, $\mathrm{WS}_{2}$ indicates the seamless high-quality in-plane suggesting monolayer one $[26,34,35]$. When heterostructure [13, 39]. Fig. 1(d) and 1(e) also considering $\mathrm{WS}_{2}$, the peak intensity ratio of demonstrate the configuration with $\mathrm{MoS}_{2}$ inside and longitudinal acoustic mode (2LA) [36] at $352 \mathrm{~cm}^{-1}$ to $\mathrm{A}_{1 \mathrm{~g}}$ mode, i.e. $\mathrm{I}_{2 \mathrm{LA}} / \mathrm{I}_{\mathrm{A} 1 \mathrm{~g}}$, is more accurate to verify the thickness than frequency difference [14]. The ratio was estimated to be $\sim 2$, in agreement with monolayer $\mathrm{WS}_{2}$ measured by $532 \mathrm{~nm}$ laser [14]. The distinct red shift of $\mathrm{E}_{2 \mathrm{~g}}$ mode (in-plane vibration) can be observed, resulted from alloying effect [37] in the lateral heterojunctions. Notably, this similar behavior were also observed in the the edges [14]. Both Raman and PL mapping vertical heterojunctions, caused by dielectric suggest a sharp and well-stitched interface between screening and interlayer coupling [38]. $\mathrm{MoS}_{2}$ and $\mathrm{WS}_{2}[14,40]$. The thickness and surface Furthermore, the Raman mapping result in Fig. 1(c) morphology were measured by atomic force with the blue region of $\mathrm{MoS}_{2}$ and the red region of microscope (AFM) with trapping-mode.

(a)
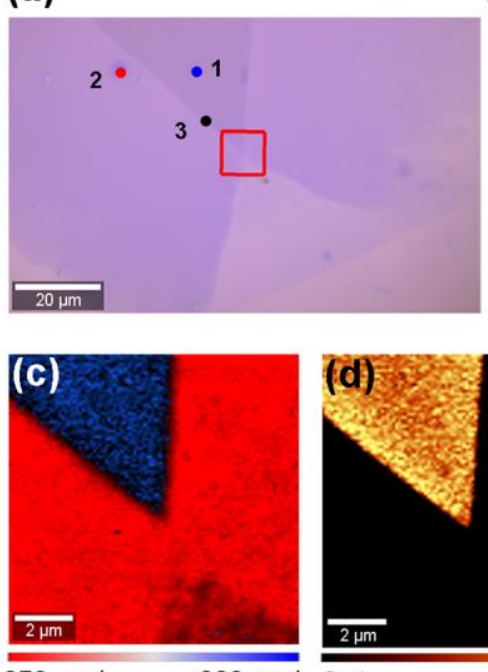
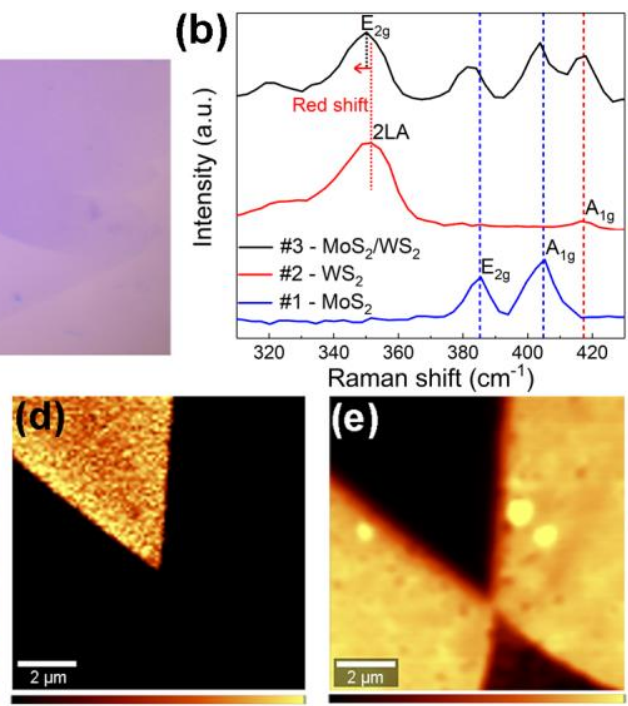

$5300 \mathrm{cts} 0 \mathrm{cts}$ (f)
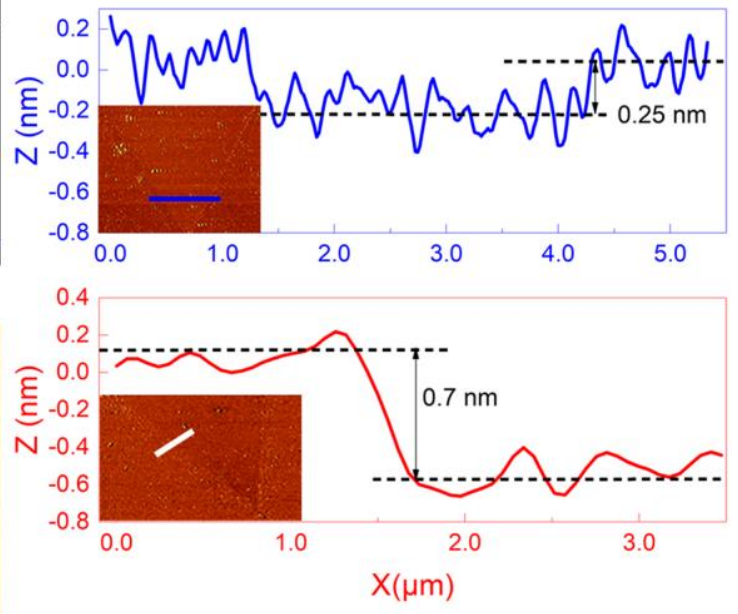

Fig. 1 Material characterization results of the as-grown lateral monolayer $\mathrm{MoS}_{2} / \mathrm{WS}_{2}$ heterostructure. (a) The optical image of the lateral monolayer $\mathrm{MoS}_{2} / \mathrm{WS}_{2}$ heterojunction. (b) The Raman spectrum obtained from the site marked with 1, 2 and 3 in (a), respectively. The Raman mapping image (c), PL mapping images of MoS2 region (d) and $\mathrm{WS}_{2}$ region (e) from the red framed 
area in (a). The corresponding false-color bar is inserted at the bottom of (c)-(e). The corresponding cross-sectional height profile of the blue (between $\mathrm{WS}_{2}$ and $\mathrm{MoS}_{2}$ ) and white (between $\mathrm{WS}_{2}$ and substrate) lines marked in AFM morphology image.

(a)
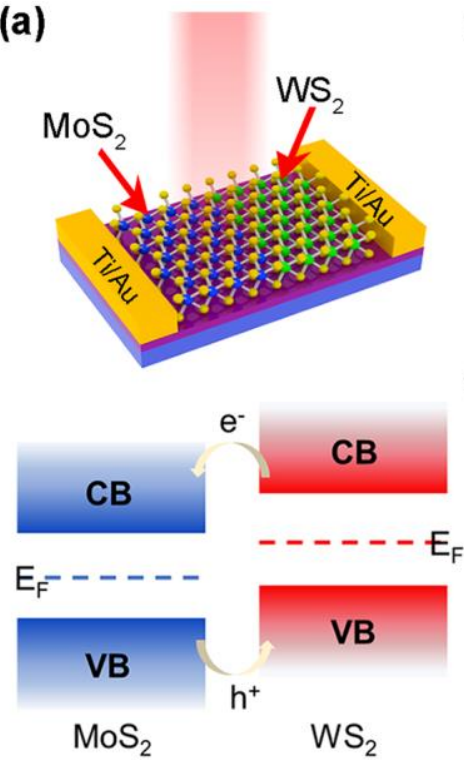

(b)
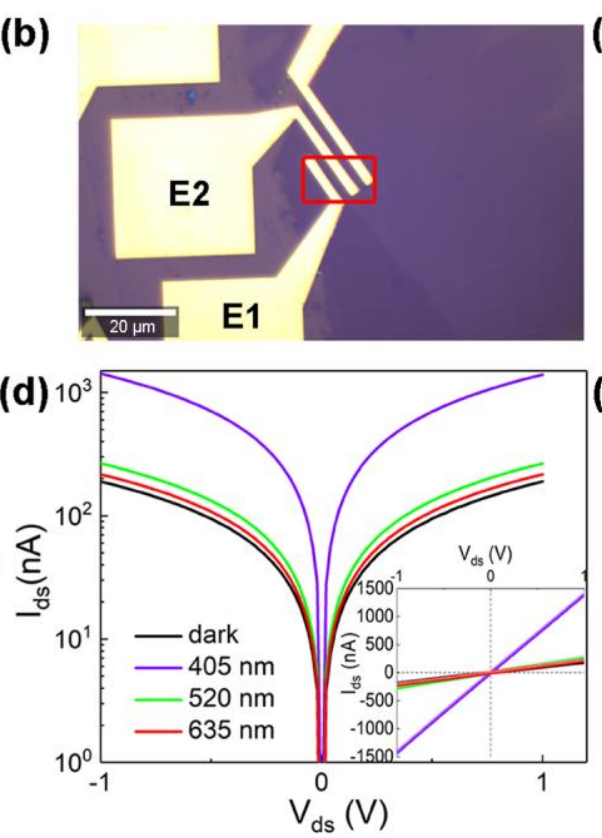

(c)
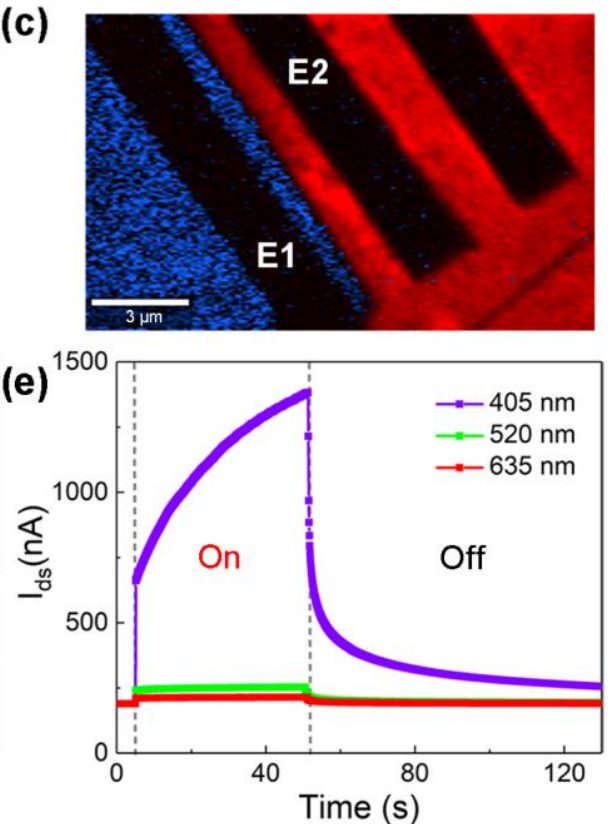

Fig. 2 Optoelectronic characteristics of the photodetector. (a) The schematic diagram and proposed band alignment of the photodetector. The optical image (b) and corresponding combined Raman mapping (c) of the photodetector. E1 and E2 represent the source and drain electrodes of the measured device. The semi-logarithmic (d) and linear (inset of (d)) I-V characteristics and the photoswitching characteristics (e) of the photodetector.

Note that few grain boundaries resulting in charge carrier scatting [41] are observed in material inside but edges indicating better electrical transport performance as shown in Fig. 1(f) [14, 42]. The thickness of $\mathrm{WS}_{2}$ outside is $\sim 0.7 \mathrm{~nm}$ (bottom) consistent with CVD-grown $\mathrm{WS}_{2}$ monolayer reported previously [43], and the height difference between $\mathrm{WS}_{2}$ and $\mathrm{MoS}_{2}$ is about $0.25 \mathrm{~nm}$ (top), implying monolayered $\mathrm{MoS}_{2}$ [43]. Overall, the above material characterization results can demonstrate the lateral monolayer $\mathrm{MoS}_{2} / \mathrm{WS}_{2}$ heterojunction with the sharp interface.

Photodetectors were fabricated using an EBL system based on the lateral $\mathrm{MoS}_{2} / \mathrm{WS}_{2}$ heterojunction. Fig. 2(a) exhibits the schematic diagram (top) of the lateral heterojunction device and corresponding type-II band alignment (bottom). Accordingly, electrons and holes are transferred and confined in $\mathrm{MoS}_{2}$ and $\mathrm{WS}_{2}$ region through the interface, respectively, achieving the photoelectric conversion $[13,18,21,44]$. We attribute this to the photogating effect, such as a special case of photoconductive effect [45]. The photogating effect can work as a local photogate modulating channel conductance [46]. The optical image of the device with the effective device area of $\sim 40 \mu \mathrm{m}^{2}$ is 

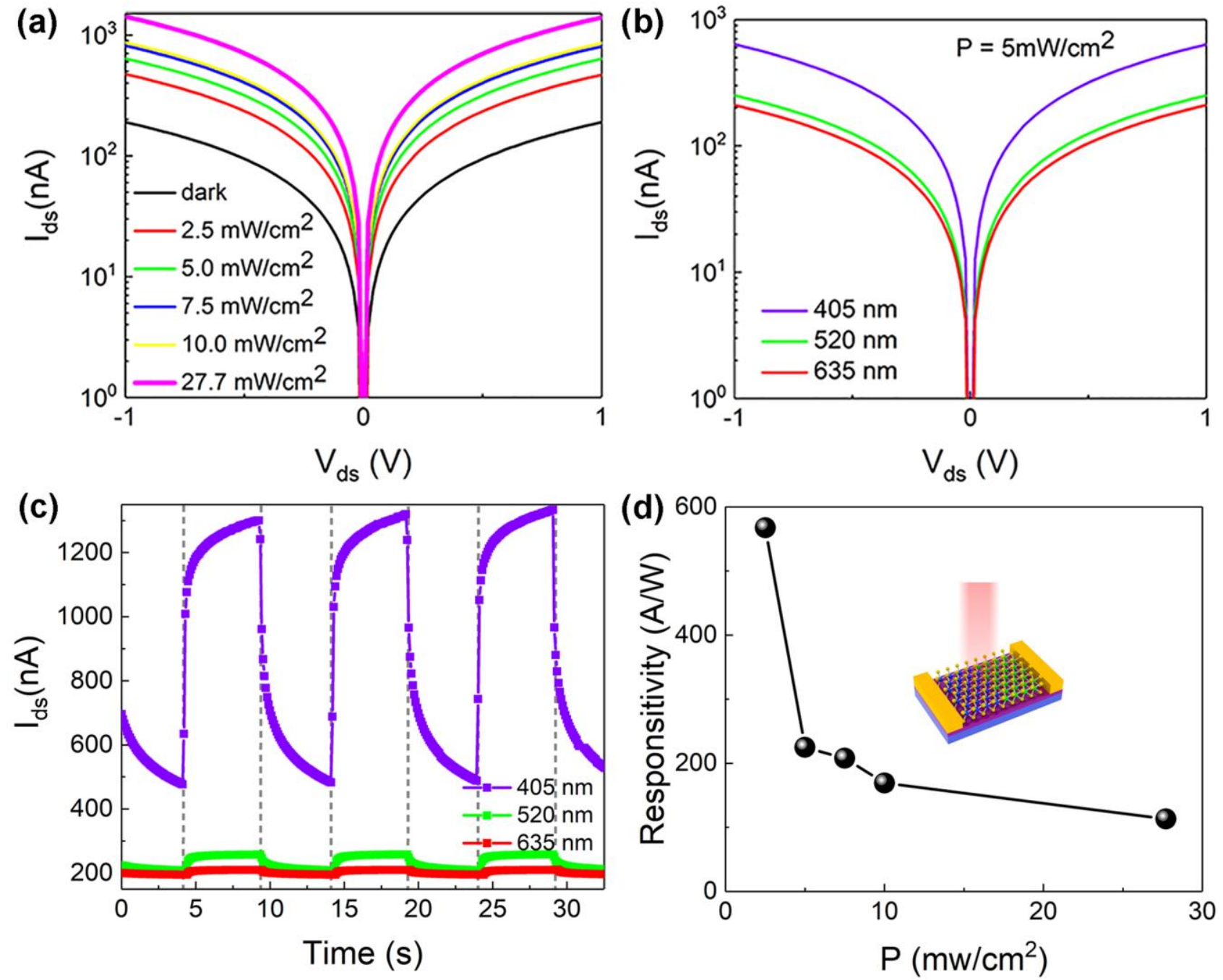

Fig. 3 Photoresponse behavior of the photodetector. The I-V characteristics under different 405 nm laser power densities (a) and under different incident wavelengths of $5 \mathrm{~mW} / \mathrm{cm}^{2}$ (b). (c) The time-resolved photoresponse excited by the periodic on/off switching of incident light. (d) The extracted R (green sphere) as a function of laser power densities.
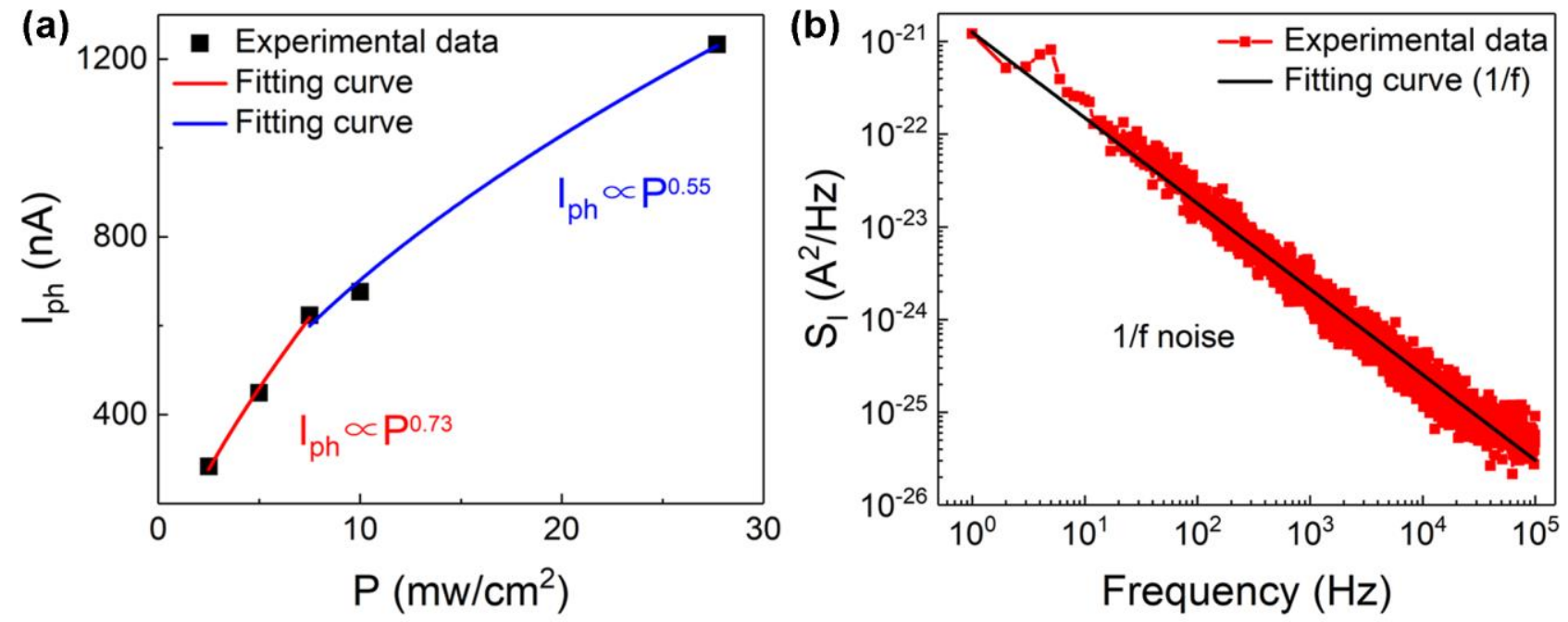

Fig. 4 (a) The plot of $\mathrm{I}_{\mathrm{ph}}$ versus laser power densities. (b) The current power spectrum ( $\left.\mathrm{S}_{\mathrm{I}}\right)$ under different frequencies.

described in Fig. 2(b) with E1 and E2 electrodes as out the heterojunction configuration, combined the source and drain electrodes. In order to figure Raman mapping was carried out (Fig. 2(c)), 
indicating the channel materials of lateral densities are depicted in Fig. 3(a). As expected, $\mathrm{MoS}_{2} / \mathrm{WS}_{2}$ heterojunction between the measured photocurrent is enlarged as the laser power densities source and drain electrodes (E1 and E2) [24]. The increase due to more induced photogenerated blue, red and dark sections are $\mathrm{MoS}_{2}, \mathrm{WS}_{2}$ and carriers [58]. Fig. 3(b) shows the I-V curves with metal electrodes, respectively. Fig. 2(d) shows the the same laser power density but different incident semi-logarithmic output characteristic curves of the lateral heterojunction under visible light with 405 $\mathrm{nm}, 520 \mathrm{~nm}$ and $635 \mathrm{~nm}$, respectively. The inset in Fig. 2(d) reveals a linear I-V relationship between the channel and the electrodes [47-52]. The linear I$\mathrm{V}$ character is conducive to achieving high responsivity but poor sensitivity of photodetectors due to a high dark current [53]. Additionally, the $\mathrm{I}_{\mathrm{ph}}$ (i.e. Ilight - $\left.\mathrm{I}_{\mathrm{dark}}\right)$ of the photodetector increases to 12.5 times of that before thermal annealing, which maybe ascribe to decreased contact resistance [42, 54], removal of defects [55] and improved electrical conductivities [56]. Fig. 2(e) depicts the photoswitching characteristics excited by the above wavelengths. The transient current rises rapidly when the light is on and drops as soon as the light is off, implying this photodetector can serve as a prompt light-activated switch [57].

The semi-logarithmic output characteristics with the same wavelength but varied laser power 
defective states [63]. Therefore, the response time including rise time and fall time was sacrificed by photogating effect because of the long-lived charge trapping processes [53]. Indeed, the figures of merit of the photosensitive devices are mainly responsibility (R) and detectivity (D*). R is calculated by the relations of

$$
R=I_{p h} /(P \cdot S)
$$

where $\mathrm{P}$ and $\mathrm{S}$ are laser power density and effective device area, respectively [58]. Fig. 3(d) shows the corresponding values of $\mathrm{R}$ of the photodetector under different laser power densities. The champion $\mathrm{R}$ reaches up to $\sim 567.6 \mathrm{~A} / \mathrm{W}$ delivering the competitive performance parameter. The high $\mathrm{R}$ is attributed to the suppressed photocarrier recombination in the heterostructure together with electron trapping in the $\mathrm{MoS}_{2}$ region presumably [19]. The decreased $\mathrm{R}$ as the laser power density increased reveals the photogating effect in the photodetector further [64].

Moreover, photocurrent and laser power density follow the power-law equation:

$$
I_{p h}=A P^{\alpha}
$$

where $\mathrm{A}$ is a constant and $0<\alpha<1$. The value of $\alpha$, obtained by fitting the curve of $\mathrm{I}_{\mathrm{ph}}$ versus $\mathrm{P}$ in Fig. 4(a), is related to the process of carrier capture, recombination and transfer $[65,66]$. The sublinear relation between $\mathrm{I}_{\mathrm{ph}}$ and $\mathrm{P}$ suggests the presence of the photogating effect in the device further [61]. The higher value of $\alpha$ (such as $\sim 0.73$ ) can be obtained when the lower power densities are applied due to reduced photocarrier recombination and the interactions between carriers $[66,67]$. In contrast, higher power densities can result in a degraded $\alpha$ value of $\sim 0.55$ because of stronger recombination losses and more trap states [68]. The precondition of the calculated $\mathrm{D}^{*}$ via the equation

$$
D^{*}=R\left(S / 2 e I_{d a r k}\right)^{1 / 2}
$$

is that the photodetectors are limited by shot noise as the main noise source $[45,62,69]$. In order to further evaluate $D^{*}$ more accurately, the noise current obtained in Fig. 4(b) is measured under different frequencies [65]. Fig. 4(b) shows the typical 1/f noise [70] in our photodetectors, which is significant impediment to semiconductor industry from new materials. This kind of noise is mainly resulted from the charged impurities and trapping sites in the conductive channel $[53,71]$. A higher material quality and small structural defect density are desired for reducing the $1 / f$ noise [72]. 
According to the formula of

$$
D^{*}=R(S \Delta f)^{1 / 2} / I_{\text {noise }}
$$

where $\Delta \mathrm{f}$ and $\mathrm{I}_{\text {noise }}$ are measurement bandwidth and noise current [70], the detectivity of the photodetector is about $7.17 \times 10^{11}$ Jones. The high $\mathrm{R}$ and $\mathrm{D}^{*}$ of our photodetectors show great potential in optoelectronic devices.

\section{Conclusions}

In summary, a high-performance photodetector was developed based on the lateral monolayer $\mathrm{MoS}_{2} / \mathrm{WS}_{2}$ heterojunction. The size of the channel materials grown by the one-step liquid-phase CVD method reaches up to millimeter scale. Moreover, the high-quality channel materials with good uniformity and sharp interface were examined by systematic material characterizations and subsequent device measurements. Particularly, high responsivity of 567.6 A/W and detectivity of $\sim 10^{11}$ Jones are achieved for the photodetectors attributing to the photogating effect. The performance of the proposed lateral $\mathrm{MoS}_{2} / \mathrm{WS}_{2}$ heterojunction photodetectors is better than or comparable to the reported work [21, 58, 67, 69, 73-75]. In addition, we suppose the undesired 1/f noise arising from the trapping/detrapping of charge carriers maybe further reduced by high-quality and defect-less channel material. The facile one-step liquid-phase CVD growth and excellent optoelectronic performance of the photodetectors can motivate further research regarding optoelectronic devices based on lateral heterostructures.

\section{Acknowledgements}

We acknowledge engineer Wanghua $\mathrm{Wu}$ for helping PL data analysis.

\section{Authors' contributions}

C. L. and H. X. conceived and designed the reasearch project. J. Z. prepared the material. C. L. fabricated the device and performed the optical characteristics. C. L. and W. D. carried out the optoelectronic measurements. C. L. analyzed the data and wrote the manuscript with the contibution form Y. H. and Z. Z.. H. X. and G. Z. supervised the project and modified manuscript.

\section{Funding}

This work was financially supported by the National Natural Science Foundation of China (G0562011530131), the National Key Research and Development Program of China (2019YFB2203400), the "111 Project" (B20030), the UESTC Shared Research Facilities of Electromagnetic Wave and Matter Interaction (Y0301901290100201), the Fundamental Research Funds for the Central Universities (ZYGX2019Z018), International Postdoctoral Exchange Fellowship Program (Talent-Introduction Program), China Postdoctoral Science Foundation (244125) and the Innovation Group Project of Sichuan Province (20CXTD0090).

\section{Availability of data and materials}

The datasets supporting the conclusions of this article are included in the article.

\section{Declaration}

\section{Competing interests}

The authors declasre they have no competing interests.

\section{References}

1. Liu, C., Chen, H., Wang, S., Liu, Q., Jiang, Y.-G., Zhang, D. W., Liu, M., and Zhou, P. (2020) Two-dimensional materials for next-generation computing technologies. Nat. Nanotechnol. 15(7); 545-557

2. Wu, G., Tian, B., Liu, L., Lv, W., Wu, S., Wang, X., Chen, Y., Li, J., Wang, Z., Wu, S., Shen, H., Lin, T., Zhou, P., Liu, Q., Duan, C., Zhang, S., Meng, X., Wu, S., Hu, W., Wang, X., Chu, J., and Wang, J. (2020) Programmable transition metal dichalcogenide homojunctions controlled by nonvolatile ferroelectric domains. Nat. Electron. 3(1); 43-50 
3. Xu, H., Ren, A., Wu, J., and Wang, Z. (2020) Recent Advances in 2D MXenes for Photodetection. Adv. Funct. Mater. 30(24); 2000907

4. Lan, Y., Xia, L.-X., Huang, T., Xu, W., Huang, G.-F., Hu, W., and Huang, W.-Q. (2020) Strain and Electric Field Controllable Schottky Barriers and Contact Types in Graphene-MoTe2 van der Waals Heterostructure. Nanoscale Res. Lett. 15(1); 180

5. Yu, J., Zhong, J., Kuang, X., Zeng, C., Cao, L., Liu, Y., and Liu, Z. (2020) Dynamic Control of High-Range Photoresponsivity in a Graphene Nanoribbon Photodetector. Nanoscale Res. Lett. 15(1); 124

6. Xu, H., Han, X., Dai, X., Liu, W., Wu, J., Zhu, J., Kim, D., Zou, G., Sablon, K. A., Sergeev, A., Guo, Z., and Liu, H. (2018) High Detectivity and Transparent Few-Layer $\mathrm{MoS}_{2} /$ Glassy-Graphene Heterostructure Photodetectors. Adv. Mater. 30(13); 1706561

7. Xu, H., Han, X., Liu, W., Liu, P., Fang, H., Li, X., Li, Z., Guo, J., Xiang, B., Hu, W., Parkin, I. P., Wu, J., Guo, Z., and Liu, H. (2020) Ambipolar and Robust $\mathrm{WSe}_{2}$ FieldEffect Transistors Utilizing Self-Assembled Edge Oxides. Adv. Mater. Interfaces 7(1); 1901628

8. Xu, H., Zhu, J., Zou, G., Liu, W., Li, X., Li, C., Ryu, G. H., Xu, W., Han, X., Guo, Z., Warner, J. H., Wu, J., and Liu, H. (2020) Spatially Bandgap-Graded $\mathrm{MoS}_{2(1-x)} \mathrm{Se}_{2 \mathrm{x}}$ Homojunctions for Self-Powered Visible-Near-Infrared Phototransistors. Nano-Micro. Lett. 12(26

9. Du, W., Yu, P., Zhu, J., Li, C., Xu, H., Zou, J., Wu, C., Wen, Q., Ji, H., Liu, T., Li, Y., Zou, G., Wu, J., and Wang, Z. M. (2020) An ultrathin $\mathrm{MoSe}_{2}$ photodetector with near-perfect absorption. Nanotechnology 31(22); 225201

10. Du, W., Li, C., Sun, J., Xu, H., Yu, P., Ren, A., Wu, J., and Wang, Z. (2020) Nanolasers Based on 2D Materials. Laser Photonics Rev. 14(12); 2000271

11. Xie, Y., Wu, E., Geng, G., Zhang, D., Hu, X., and Liu, J. (2021) Gate-tunable van der Waals heterostructure based on semimetallic $\mathrm{WTe}_{2}$ and semiconducting $\mathrm{MoTe}_{2}$. Appl. Phys. Lett. 118(13); 133103

12. Ning, J., Zhou, Y., Zhang, J., Lu, W., Dong, J., Yan, C., Wang, D., Shen, X., Feng, X., Zhou, H., and Hao, Y. (2020) Self-driven photodetector based on a GaSe/MoSe selenide van der Waals heterojunction with the hybrid contact. Appl. Phys. Lett. 117(16); 163104

13. Cai, Z., Liu, B., Zou, X., and Cheng, H.-M. (2018) Chemical Vapor Deposition Growth and Applications of Two-Dimensional Materials and Their Heterostructures. Chem. Rev. 118(13); 6091-6133

14. Shen, P. C., Engineering, M. I. o. T. D. o. E., and Science, C. (2017) Large-area CVD Growth of Two-dimensional Transition Metal Dichalcogenides and Monolayer $\mathrm{MoS}_{2}$ and $\mathrm{WS}_{2} \quad$ Metal-oxide-semiconductor Field-effect Transistors, Massachusetts Institute of Technology, Department of Electrical Engineering and Computer Science.

15. Wang, X., Lu, Y., Zhang, J., Zhang, S., Chen, T., Ou, Q., and Huang, J. (2021) Highly Sensitive Artificial Visual Array Using Transistors Based on Porphyrins and Semiconductors. Small 17(2); 2005491
16. Zhou, F., Zhou, Z., Chen, J., Choy, T. H., Wang, J., Zhang, N., Lin, Z., Yu, S., Kang, J., Wong, H. S. P., and Chai, Y. (2019) Optoelectronic resistive random access memory for neuromorphic vision sensors. Nat. Nanotechnol. 14(8); 776-782

17. Wang, P., and Hu, W. (2019) Atomic Layered 2d/3d Heterostructure for Sensitive Photodetection, in 2019 18th International Conference on Optical Communications and Networks (ICOCN), pp 1-3.

18. Liu, Y., Zhang, S., He, J., Wang, Z. M., and Liu, Z. (2019) Recent Progress in the Fabrication, Properties, and Devices of Heterostructures Based on 2D Materials. Nano-Micro. Lett. 11(1); 13

19. Nalwa, H. S. (2020) A review of molybdenum disulfide $\left(\mathrm{MoS}_{2}\right)$ based photodetectors: from ultra-broadband, selfpowered to flexible devices. RSC Adv. 10(51); 3052930602

20. Neupane, M. R., Ruzmetov, D., Burke, R., Birdwell, A. G., Taylor, D., Chin, M., Regan, T. O., Crowne, F., Nichols, B., Shah, P., Byrd, E., and Ivanov, T. (2018) Challenges and opportunities in integration of 2D materials on 3D substrates: Materials and device perspectives, in 2018 76th Device Research Conference $(D R C)$, pp 1-2.

21. Wu, W., Zhang, Q., Zhou, X., Li, L., Su, J., Wang, F., and Zhai, T. (2018) Self-powered photovoltaic photodetector established on lateral monolayer $\mathrm{MoS}_{2}-\mathrm{WS}_{2}$ heterostructures. Nano Energy 51(45-53

22. Wang, W., He, J., Cao, Y., Kong, L., Zheng, X., Wu, Y., Chen, X., Li, S., Wu, Z., and Kang, J. (2017) Nonuniform Effect of Carrier Separation Efficiency and Light Absorption in Type-II Perovskite Nanowire Solar Cells. Nanoscale Res. Lett. 12(

23. Cheng, H., Huang, Y., and Duan, X. (2016) Vertically stacked heterostructures for tunable photonic devices from 2D materials to hybrid perovskites, in 2016 Conference on Lasers and Electro-Optics (CLEO), pp 1-2.

24. Jia, S., Jin, Z., Zhang, J., Yuan, J., Chen, W., Feng, W., Hu, P., Ajayan, P. M., and Lou, J. (2020) Lateral Monolayer $\mathrm{MoSe}_{2}-\mathrm{WSe}_{2} \mathrm{p}-\mathrm{n}$ Heterojunctions with Giant Built-In Potentials. Small 16(34); 2002263

25. Zhou, Z., Zhang, Y., Zhang, X., Niu, X., Wu, G., and Wang, J. (2020) Suppressing photoexcited electron-hole recombination in $\mathrm{MoSe}_{2} / \mathrm{WSe}_{2}$ lateral heterostructures via interface-coupled state engineering: a time-domain ab initio study. J. Mater. Chem. A 8(39); 20621-20628

26. Zhu, J., Li, W., Huang, R., Ma, L., Sun, H., Choi, J.-H., Zhang, L., Cui, Y., and Zou, G. (2020) One-Pot Selective Epitaxial Growth of Large $\mathrm{WS}_{2} / \mathrm{MoS}_{2}$ Lateral and Vertical Heterostructures. J. Am. Chem. Soc. 142(38); 1627616284

27. Akinwande, D., Huyghebaert, C., Wang, C.-H., Serna, M. I., Goossens, S., Li, L.-J., Wong, H. S. P., and Koppens, F. H. L. (2019) Graphene and two-dimensional materials for silicon technology. Nature 573(7775); 507-518

28. Yang, W., Kawai, H., Bosman, M., Tang, B., Chai, J., Tay, W. L., Yang, J., Seng, H. L., Zhu, H., Gong, H., Liu, H., Goh, K. E. J., Wang, S., and Chi, D. (2018) Interlayer interactions in 2D $\quad \mathrm{WS}_{2} / \mathrm{MoS}_{2}$ heterostructures 
monolithically grown by in situ physical vapor deposition. Nanoscale 10(48); 22927-22936

29. Zhang, X., Huangfu, L., Gu, Z., Xiao, S., Zhou, J., Nan, H., Gu, X., and Ostrikov, K. (2021) Controllable Epitaxial Growth of Large-Area $\mathrm{MoS}_{2} / \mathrm{WS}_{2} \quad$ Vertical Heterostructures by Confined-Space Chemical Vapor Deposition. Small 17(18); 2007312

30. Kang, J., Tongay, S., Zhou, J., Li, J., and Wu, J. (2013) Band offsets and heterostructures of two-dimensional semiconductors. Appl. Phys. Lett. 102(1); 012111

31. Chen, K., Wan, X., Wen, J., Xie, W., Kang, Z., Zeng, X., Chen, H., and Xu, J.-B. (2015) Electronic Properties of $\mathrm{MoS}_{2}-\mathrm{WS}_{2}$ Heterostructures Synthesized with Two-Step Lateral Epitaxial Strategy. ACS Nano 9(10); 9868-9876

32. Zavabeti, A., Jannat, A., Zhong, L., Haidry, A. A., Yao, Z., and Ou, J. Z. (2020) Two-Dimensional Materials in Large-Areas: Synthesis, Properties and Applications. Nano-Micro. Lett. 12(1); 66

33. Liu, H., and Xue, Y. (2021) Van Der Waals Epitaxial Growth and Phase Transition of Layered $\mathrm{FeSe}_{2}$ Nanocrystals. Adv. Mater. 33(17); 2008456

34. Lee, H. S., Min, S.-W., Chang, Y.-G., Park, M. K., Nam, T., Kim, H., Kim, J. H., Ryu, S., and Im, S. (2012) $\mathrm{MoS}_{2}$ Nanosheet Phototransistors with Thickness-Modulated Optical Energy Gap. Nano Lett. 12(7); 3695-3700

35. Kim, M., Seo, J., Kim, J., Moon, J. S., Lee, J., Kim, J.-H., Kang, J., and Park, H. (2021) High-Crystalline Monolayer Transition Metal Dichalcogenides Films for Wafer-Scale Electronics. ACS Nano 15(2); 3038-3046

36. Berkdemir, A., Gutiérrez, H. R., Botello-Méndez, A. R., Perea-López, N., Elías, A. L., Chia, C.-I., Wang, B., Crespi, V. H., López-Urías, F., Charlier, J.-C., Terrones, H., and Terrones, M. (2013) Identification of individual and few layers of $\mathrm{WS}_{2}$ using Raman Spectroscopy. Sci. Rep. 3(1); 1755

37. Sahoo, P. K., Memaran, S., Xin, Y., Balicas, L., and Gutiérrez, H. R. (2018) One-pot growth of twodimensional lateral heterostructures via sequential edgeepitaxy. Nature 553(7686); 63-67

38. Liang, L., and Meunier, V. (2014) First-principles Raman spectra of $\mathrm{MoS}_{2}, \mathrm{WS}_{2}$ and their heterostructures. Nanoscale 6(10); 5394-5401

39. Zhang, Z., Chen, P., Duan, X., Zang, K., Luo, J., and Duan, X. (2017) Robust epitaxial growth of twodimensional heterostructures, multiheterostructures, and superlattices. Science 357(6353); 788-792

40. Ye, K., Liu, L., Liu, Y., Nie, A., Zhai, K., Xiang, J., Wang, B., Wen, F., Mu, C., Zhao, Z., Gong, Y., Liu, Z., and Tian, Y. (2019) Lateral Bilayer $\mathrm{MoS}_{2}-\mathrm{WS}_{2}$ Heterostructure Photodetectors with High Responsivity and Detectivity. Adv. Opt. Mater. 7(20); 1900815

41. Tsen, A. W., Brown, L., Levendorf, M. P., Ghahari, F., Huang, P. Y., Havener, R. W., Ruiz-Vargas, C. S., Muller, D. A., Kim, P., and Park, J. (2012) Tailoring Electrical Transport Across Grain Boundaries in Polycrystalline Graphene. Science 336(6085); 1143-1146

42. Wu, M., Xiao, Y., Zeng, Y., Zhou, Y., Zeng, X., Zhang, L., and Liao, W. (2020) Synthesis of two-dimensional transition metal dichalcogenides for electronics and optoelectronics. InfoMat 3(4); 362-396
43. Xiao, J., Zhang, Y., Chen, H., Xu, N., and Deng, S. (2018) Enhanced Performance of a Monolayer $\mathrm{MoS}_{2} / \mathrm{WSe}_{2}$ Heterojunction as a Photoelectrochemical Cathode. NanoMicro. Lett. 10(4); 60

44. Cheng, K., Guo, Y., Han, N., Su, Y., Zhang, J., and Zhao, J. (2017) Lateral heterostructures of monolayer group-IV monochalcogenides: band alignment and electronic properties. J. Mater. Chem. C 5(15); 3788-3795

45. Shin, G. H., Park, C., Lee, K. J., Jin, H. J., and Choi, S.-Y. (2020) Ultrasensitive Phototransistor Based on $\mathrm{WSe}_{2}-$ $\mathrm{MoS}_{2}$ van der Waals Heterojunction. Nano Lett. 20(8); 5741-5748

46. Ramos, M., Carrascoso, F., Frisenda, R., Gant, P., MañasValero, S., Esteras, D. L., Baldoví, J. J., Coronado, E., Castellanos-Gomez, A., and Calvo, M. R. (2021) Ultrabroad spectral photo-response in $\mathrm{FePS}_{3}$ air-stable devices. npj 2D Mater. Appl. 5(1); 19

47. Shen, P.-C., Su, C., Lin, Y., Chou, A.-S., Cheng, C.-C., Park, J.-H., Chiu, M.-H., Lu, A.-Y., Tang, H.-L., Tavakoli, M. M., Pitner, G., Ji, X., Cai, Z., Mao, N., Wang, J., Tung, V., Li, J., Bokor, J., Zettl, A., Wu, C.-I., Palacios, T., Li, L.-J., and Kong, J. (2021) Ultralow contact resistance between semimetal and monolayer semiconductors. Nature 593(7858); 211-217

48. Wang, Y., Kim, J. C., Wu, R. J., Martinez, J., Song, X., Yang, J., Zhao, F., Mkhoyan, A., Jeong, H. Y., and Chhowalla, M. (2019) Van der Waals contacts between three-dimensional metals and two-dimensional semiconductors. Nature 568(7750); 70-74

49. Piprek, G., and Piprek, J. (2003) Semiconductor Optoelectronic Devices: Introduction to Physics and Simulation, Elsevier Science, USA.

50. Sze, S. M., and Ng, K. K. (2006) Physics of Semiconductor Devices, Wiley.

51. Fashandi, H., Dahlqvist, M., Lu, J., Palisaitis, J., Simak, S. I., Abrikosov, I. A., Rosen, J., Hultman, L., Andersson, M., Lloyd Spetz, A., and Eklund, P. (2017) Synthesis of $\mathrm{Ti}_{3} \mathrm{AuC}_{2}, \quad \mathrm{Ti}_{3} \mathrm{Au}_{2} \mathrm{C}_{2}$ and $\mathrm{Ti}_{3} \mathrm{IrC}_{2}$ by noble metal substitution reaction in $\mathrm{Ti}_{3} \mathrm{SiC}_{2}$ for high-temperaturestable Ohmic contacts to SiC. Nat. Mater. 16(8); 814-818

52. Bittle, E. G., Basham, J. I., Jackson, T. N., Jurchescu, O. D., and Gundlach, D. J. (2016) Mobility overestimation due to gated contacts in organic field-effect transistors. Nat. Commun. 7(1); 10908

53. Kufer, D., and Konstantatos, G. (2015) Highly Sensitive, Encapsulated $\mathrm{MoS}_{2}$ Photodetector with Gate Controllable Gain and Speed. Nano Lett. 15(11); 7307-7313

54. Zhu, Y., Cao, W., Fan, Y., Deng, Y., and Xu, C. (2014) Effects of rapid thermal annealing on ohmic contact of AlGaN/GaN HEMTs. J. Semicond. 35(2); 026004

55. Park, C.-S. (2018) Disorder induced transition of electrical properties of graphene by thermal annealing. Results Phys. 9(1534-1536

56. Yang, Q., Beers, M. H., Mehta, V., Gao, T., and Parkinson, D. (2017) Effect of Thermal Annealing on the Electrical Conductivity of Copper-Tin Polymer Composites. ACS Appl. Mater. Interfaces 9(1); 958-964

57. Pradhan, B., Das, S., Li, J., Chowdhury, F., Cherusseri, J., Pandey, D., Dev, D., Krishnaprasad, A., Barrios, E., Towers, A., Gesquiere, A., Tetard, L., Roy, T., and 
Thomas, J. (2020) Ultrasensitive and ultrathin phototransistors and photonic synapses using perovskite quantum dots grown from graphene lattice. Sci. Adv. 6(7); eaay5225

58. Yang, T., Zheng, B., Wang, Z., Xu, T., Pan, C., Zou, J., Zhang, X., Qi, Z., Liu, H., Feng, Y., Hu, W., Miao, F., Sun, L., Duan, X., and Pan, A. (2017) Van der Waals epitaxial growth and optoelectronics of large-scale $\mathrm{WSe}_{2} / \mathrm{SnS}_{2}$ vertical bilayer $\mathrm{p}-\mathrm{n}$ junctions. Nat. Commun. 8(1); 1906

59. Bissett, M., Worrall, S., Kinloch, I., and Dryfe, R. (2016) Comparison of Two-Dimensional Transition Metal Dichalcogenides for Electrochemical Supercapacitors. Electrochim. Acta. 201(30-37

60. Liu, H.-L., Shen, C.-C., Su, S.-H., Hsu, C.-L., Li, M.-Y., and Li, L.-J. (2014) Optical properties of monolayer transition metal dichalcogenides probed by spectroscopic ellipsometry. Appl. Phys. Lett. 105(20); 201905

61. Fang, H., and Hu, W. (2017) Photogating in Low Dimensional Photodetectors. Adv. Sci. 4(12); 1700323

62. Zhao, Q., Wang, W., Carrascoso-Plana, F., Jie, W., Wang, T., Castellanos-Gomez, A., and Frisenda, R. (2020) The role of traps in the photocurrent generation mechanism in thin InSe photodetectors. Mater. Horiz. 7(1); 252-262

63. Jiang, J., Meng, F., Cheng, Q., Wang, A., Chen, Y., Qiao, J., Pang, J., Xu, W., Ji, H., Zhang, Y., Zhang, Q., Wang, S., Feng, X., Gu, L., Liu, H., and Han, L. (2020) Low Lattice Mismatch InSe-Se Vertical Van der Waals Heterostructure for High-performance Transistors via Strong Fermi-Level Depinning. Small Methods 4(8); 2000238

64. Lan, H.-Y., Hsieh, Y.-H., Chiao, Z.-Y., Jariwala, D., Shih, M.-H., Yen, T.-J., Hess, O., and Lu, Y.-J. (2021) GateTunable Plasmon-Enhanced Photodetection in a Monolayer $\mathrm{MoS}_{2}$ Phototransistor with Ultrahigh Photoresponsivity. Nano Lett.

65. Zhao, Z., Wu, D., Guo, J., Wu, E., Jia, C., Shi, Z., Tian, Y., Li, X., and Tian, Y. (2019) Synthesis of large-area 2D $\mathrm{WS}_{2}$ films and fabrication of a heterostructure for selfpowered ultraviolet photodetection and imaging applications. J. Mater. Chem. C 7(39); 12121-12126

66. Cao, R., Xu, J., Shi, S., Chen, J., Liu, D., Bu, Y., Zhang, X., Yin, S., and Li, L. (2020) High-performance selfpowered ultraviolet photodetectors based on mixeddimensional heterostructure arrays formed from $\mathrm{NiO}$ nanosheets and $\mathrm{TiO}_{2}$ nanorods. J. Mater. Chem. C 8(28); 9646-9654

67. Zeng, L., Tao, L., Tang, C., Zhou, B., Long, H., Chai, Y., Lau, S. P., and Tsang, Y. H. (2016) High-responsivity UV-Vis Photodetector Based on Transferable $\mathrm{WS}_{2}$ Film Deposited by Magnetron Sputtering. Sci. Rep. 6(20343

68. Natali, D., and Caironi, M. (2016) 7 - Organic photodetectors, in Photodetectors (Nabet, B., Ed.), pp 195-254, Woodhead Publishing.

69. Ren, A., Zou, J., Lai, H., Huang, Y., Yuan, L., Xu, H., Shen, K., Wang, H., Wei, S., Wang, Y., Hao, X., Zhang, J., Zhao, D., Wu, J., and Wang, Z. (2020) Direct laserpatterned MXene-perovskite image sensor arrays for visible-near infrared photodetection. Mater. Horiz. 7(7); 1901-1911
70. Schneider H, L. H. C. (2007) Quantum Well Infrared Photodetectors: Physics and Applications, Springer, Berlin.

71. Macucci, M., and Marconcini, P. (2020) Theoretical Comparison between the Flicker Noise Behavior of Graphene and of Ordinary Semiconductors. J. Sens. 2020(2850268

72. Balandin, A. A. (2013) Low-frequency $1 / \mathrm{f}$ noise in graphene devices. Nat. Nanotechnol. 8(8); 549-555

73. Zhang, D.-D., and Yu, R.-M. (2019) Perovskite-WS $\mathrm{W}_{2}$ Nanosheet Composite Optical Absorbers on Graphene as High-Performance Phototransistors. Frontiers in Chemistry 7(257)

74. Lan, C., Zhou, Z., Zhou, Z., Li, C., Shu, L., Shen, L., Li, D., Dong, R., Yip, S., and Ho, J. C. (2018) Wafer-scale synthesis of monolayer $\mathrm{WS}_{2}$ for high-performance flexible photodetectors by enhanced chemical vapor deposition. Nano Res. 11(6); 3371-3384

75. Tsai, T.-H., Liang, Z.-Y., Lin, Y.-C., Wang, C.-C., Lin, K.-I., Suenaga, K., and Chiu, P.-W. (2020) Photogating $\mathrm{WS}_{2}$ Photodetectors Using Embedded $\mathrm{WSe}_{2}$ Charge Puddles. ACS Nano 14(4); 4559-4566 